\title{
Complex Function Differentiability
}

\author{
Chanapat Pacharapokin \\ Shinshu University \\ Nagano, Japan
}

\author{
Hiroshi Yamazaki \\ Shinshu University \\ Nagano, Japan \\ Yatsuka Nakamura \\ Shinshu University \\ Nagano, Japan
}

\author{
Yasunari Shidama \\ Shinshu University \\ Nagano, Japan
}

Summary. For a complex valued function defined on its domain in complex numbers the differentiability in a single point and on a subset of the domain is presented. The main elements of differential calculus are developed. The algebraic properties of differential complex functions are shown.

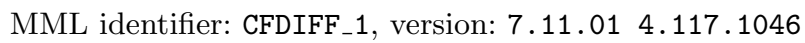

The articles [17], [18], [3], [5], [4], [8], [2], [7], [11], [6], [16], [12], [19], [9], [10], [1], [14], [15], and [13] provide the notation and terminology for this paper.

For simplicity, we use the following convention: $k, n, m$ denote elements of $\mathbb{N}, X$ denotes a set, $s_{1}, s_{2}$ denote complex sequences, $Y$ denotes a subset of $\mathbb{C}$, $f, f_{1}, f_{2}$ denote partial functions from $\mathbb{C}$ to $\mathbb{C}, r$ denotes a real number, $a, a_{1}$, $b, x, x_{0}, z, z_{0}$ denote complex numbers, and $N_{1}$ denotes an increasing sequence of naturals.

Let $I$ be a complex sequence. We say that $I$ is convergent to 0 if and only if:

(Def. 1) $I$ is non-zero and convergent and $\lim I=0$.

We now state four propositions:

(1) Let $r_{1}$ be a sequence of real numbers and $c_{1}$ be a complex sequence. If $r_{1}=c_{1}$ and $r_{1}$ is convergent, then $c_{1}$ is convergent.

(2) If $0<r$ and for every $n$ holds $s_{1}(n)=\frac{1}{n+r}$, then $s_{1}$ is convergent.

(3) If $0<r$ and for every $n$ holds $s_{1}(n)=\frac{1}{n+r}$, then $\lim s_{1}=0$.

(4) If for every $n$ holds $s_{1}(n)=\frac{1}{n+1}$, then $s_{1}$ is convergent and $\lim s_{1}=0$. 
Let us observe that there exists a complex sequence which is convergent to 0 .

Let us note that there exists a complex sequence which is constant.

Next we state four propositions:

(5) $s_{1}$ is constant iff for all $n, m$ holds $s_{1}(n)=s_{1}(m)$.

(6) For every $n$ holds $\left(s_{1} \cdot N_{1}\right)(n)=s_{1}\left(N_{1}(n)\right)$.

(7) If $s_{1}$ is constant and $s_{2}$ is a subsequence of $s_{1}$, then $s_{2}$ is constant.

(8) If $s_{1}$ is constant and $s_{2}$ is a subsequence of $s_{1}$, then $s_{1}=s_{2}$.

Let $s_{3}$ be a constant complex sequence. Note that every subsequence of $s_{3}$ is constant.

In the sequel $h$ is a convergent to 0 complex sequence and $c$ is a constant complex sequence.

Let $I$ be a partial function from $\mathbb{C}$ to $\mathbb{C}$. We say that $I$ is rest-like if and only if:

(Def. 2) $I$ is total and for every $h$ holds $h^{-1}(I \cdot h)$ is convergent and $\lim \left(h^{-1}(I\right.$. $h))=0$.

Let us mention that there exists a partial function from $\mathbb{C}$ to $\mathbb{C}$ which is rest-like.

A $\mathbb{C}$-rest is a rest-like partial function from $\mathbb{C}$ to $\mathbb{C}$.

Let $I$ be a partial function from $\mathbb{C}$ to $\mathbb{C}$. We say that $I$ is linear if and only if:

(Def. 3) $I$ is total and there exists $a$ such that for every $z$ holds $I_{z}=a \cdot z$.

One can check that there exists a partial function from $\mathbb{C}$ to $\mathbb{C}$ which is linear.

A $\mathbb{C}$-linear function is a linear partial function from $\mathbb{C}$ to $\mathbb{C}$.

We adopt the following convention: $R, R_{1}, R_{2}$ are $\mathbb{C}$-rests and $L, L_{1}, L_{2}$ are $\mathbb{C}$-linear functions.

Let us consider $L_{1}, L_{2}$. Observe that $L_{1}+L_{2}$ is linear and $L_{1}-L_{2}$ is linear.

The following propositions are true:

(9) For all $L_{1}, L_{2}$ holds $L_{1}+L_{2}$ is a $\mathbb{C}$-linear function and $L_{1}-L_{2}$ is a $\mathbb{C}$-linear function.

(10) For all $a, L$ holds $a L$ is a $\mathbb{C}$-linear function.

(11) For all $R_{1}, R_{2}$ holds $R_{1}+R_{2}$ is a $\mathbb{C}$-rest and $R_{1}-R_{2}$ is a $\mathbb{C}$-rest and $R_{1} R_{2}$ is a $\mathbb{C}$-rest.

(12) $a R$ is a $\mathbb{C}$-rest.

(13) $L_{1} L_{2}$ is rest-like.

(14) $R L$ is a $\mathbb{C}$-rest and $L R$ is a $\mathbb{C}$-rest.

Let $z_{0}$ be a complex number. A subset of $\mathbb{C}$ is called a neighbourhood of $z_{0}$ if: 
(Def. 4) There exists a real number $g$ such that $0<g$ and $\{y$; $y$ ranges over complex numbers: $\left.\left|y-z_{0}\right|<g\right\} \subseteq$ it.

Next we state three propositions:

(15) For every real number $g$ such that $0<g$ holds $\{y$; $y$ ranges over complex numbers: $\left.\left|y-z_{0}\right|<g\right\}$ is a neighbourhood of $z_{0}$.

(16) For every neighbourhood $N$ of $z_{0}$ holds $z_{0} \in N$.

(17) Let $z_{0}$ be a complex number and $N_{2}, N_{3}$ be neighbourhoods of $z_{0}$. Then there exists a neighbourhood $N$ of $z_{0}$ such that $N \subseteq N_{2}$ and $N \subseteq N_{3}$.

Let us consider $f$ and let $x_{0}$ be a complex number. We say that $f$ is differentiable in $x_{0}$ if and only if the condition (Def. 5) is satisfied.

(Def. 5) There exists a neighbourhood $N$ of $x_{0}$ such that $N \subseteq \operatorname{dom} f$ and there exist $L, R$ such that for every complex number $x$ such that $x \in N$ holds $f_{x}-f_{x_{0}}=L_{x-x_{0}}+R_{x-x_{0}}$.

Let us consider $f$ and let $z_{0}$ be a complex number. Let us assume that $f$ is differentiable in $z_{0}$. The functor $f^{\prime}\left(z_{0}\right)$ yielding a complex number is defined by the condition (Def. 6).

(Def. 6) There exists a neighbourhood $N$ of $z_{0}$ such that $N \subseteq \operatorname{dom} f$ and there exist $L, R$ such that $f^{\prime}\left(z_{0}\right)=L_{1_{\mathbb{C}}}$ and for every complex number $z$ such that $z \in N$ holds $f_{z}-f_{z_{0}}=L_{z-z_{0}}+R_{z-z_{0}}$.

Let us consider $f, X$. We say that $f$ is differentiable on $X$ if and only if:

(Def. 7) $X \subseteq \operatorname{dom} f$ and for every $x$ such that $x \in X$ holds $f\lceil X$ is differentiable in $x$.

We now state the proposition

(18) If $f$ is differentiable on $X$, then $X$ is a subset of $\mathbb{C}$.

Let $X$ be a subset of $\mathbb{C}$. We say that $X$ is closed if and only if:

(Def. 8) For every complex sequence $s_{3}$ such that rng $s_{3} \subseteq X$ and $s_{3}$ is convergent holds $\lim s_{3} \in X$.

Let $X$ be a subset of $\mathbb{C}$. We say that $X$ is open if and only if:

(Def. 9) $\quad X^{\mathrm{c}}$ is closed.

Next we state several propositions:

(19) Let $X$ be a subset of $\mathbb{C}$. Suppose $X$ is open. Let $z_{0}$ be a complex number. If $z_{0} \in X$, then there exists a neighbourhood $N$ of $z_{0}$ such that $N \subseteq X$.

(20) Let $X$ be a subset of $\mathbb{C}$. Suppose $X$ is open. Let $z_{0}$ be a complex number. Suppose $z_{0} \in X$. Then there exists a real number $g$ such that $\{y ; y$ ranges over complex numbers: $\left.\left|y-z_{0}\right|<g\right\} \subseteq X$.

(21) Let $X$ be a subset of $\mathbb{C}$. Suppose that for every complex number $z_{0}$ such that $z_{0} \in X$ there exists a neighbourhood $N$ of $z_{0}$ such that $N \subseteq X$. Then $X$ is open. 
(22) Let $X$ be a subset of $\mathbb{C}$. Then $X$ is open if and only if for every complex number $x$ such that $x \in X$ there exists a neighbourhood $N$ of $x$ such that $N \subseteq X$.

(23) Let $X$ be a subset of $\mathbb{C}, z_{0}$ be an element of $\mathbb{C}$, and $r$ be an element of $\mathbb{R}$. If $X=\left\{y ; y\right.$ ranges over complex numbers: $\left.\left|y-z_{0}\right|<r\right\}$, then $X$ is open.

(24) Let $X$ be a subset of $\mathbb{C}, z_{0}$ be an element of $\mathbb{C}$, and $r$ be an element of $\mathbb{R}$. If $X=\left\{y ; y\right.$ ranges over complex numbers: $\left.\left|y-z_{0}\right| \leq r\right\}$, then $X$ is closed.

Let us note that there exists a subset of $\mathbb{C}$ which is open.

In the sequel $Z$ denotes an open subset of $\mathbb{C}$.

Next we state two propositions:

(25) $f$ is differentiable on $Z$ iff $Z \subseteq \operatorname{dom} f$ and for every $x$ such that $x \in Z$ holds $f$ is differentiable in $x$.

(26) If $f$ is differentiable on $Y$, then $Y$ is open.

Let us consider $f, X$. Let us assume that $f$ is differentiable on $X$. The functor $f_{\lceil X}^{\prime}$ yielding a partial function from $\mathbb{C}$ to $\mathbb{C}$ is defined by:

(Def. 10) $\operatorname{dom}\left(f_{\lceil X}^{\prime}\right)=X$ and for every $x$ such that $x \in X$ holds $\left(f_{\lceil X}^{\prime}\right)_{x}=f^{\prime}(x)$.

The following propositions are true:

(27) Let given $f, Z$. Suppose $Z \subseteq \operatorname{dom} f$ and there exists $a_{1}$ such that $\operatorname{rng} f=$ $\left\{a_{1}\right\}$. Then $f$ is differentiable on $Z$ and for every $x$ such that $x \in Z$ holds $\left(f_{\lceil Z}^{\prime}\right)_{x}=0_{\mathbb{C}}$.

(28) If $s_{1}$ is non-zero, then $s_{1} \uparrow k$ is non-zero.

Let us consider $h, n$. Note that $h \uparrow n$ is convergent to 0 .

Let us consider $c, n$. Note that $c \uparrow n$ is constant.

Next we state a number of propositions:

(29) $\quad\left(s_{1}+s_{2}\right) \uparrow k=s_{1} \uparrow k+s_{2} \uparrow k$.

(30) $\quad\left(s_{1}-s_{2}\right) \uparrow k=s_{1} \uparrow k-s_{2} \uparrow k$.

(31) $s_{1}{ }^{-1} \uparrow k=\left(s_{1} \uparrow k\right)^{-1}$.

(32) $\quad\left(s_{1} s_{2}\right) \uparrow k=\left(s_{1} \uparrow k\right)\left(s_{2} \uparrow k\right)$.

(33) Let $x_{0}$ be a complex number and $N$ be a neighbourhood of $x_{0}$. Suppose $f$ is differentiable in $x_{0}$ and $N \subseteq \operatorname{dom} f$. Let given $h, c$. Suppose $\operatorname{rng} c=\left\{x_{0}\right\}$ and $\operatorname{rng}(h+c) \subseteq N$. Then $h^{-1}(f \cdot(h+c)-f \cdot c)$ is convergent and $f^{\prime}\left(x_{0}\right)=\lim \left(h^{-1}(f \cdot(h+c)-f \cdot c)\right)$.

(34) Let given $f_{1}, f_{2}, x_{0}$. Suppose $f_{1}$ is differentiable in $x_{0}$ and $f_{2}$ is differentiable in $x_{0}$. Then $f_{1}+f_{2}$ is differentiable in $x_{0}$ and $\left(f_{1}+f_{2}\right)^{\prime}\left(x_{0}\right)=$ $f_{1}^{\prime}\left(x_{0}\right)+f_{2}^{\prime}\left(x_{0}\right)$.

(35) Let given $f_{1}, f_{2}, x_{0}$. Suppose $f_{1}$ is differentiable in $x_{0}$ and $f_{2}$ is differentiable in $x_{0}$. Then $f_{1}-f_{2}$ is differentiable in $x_{0}$ and $\left(f_{1}-f_{2}\right)^{\prime}\left(x_{0}\right)=$ $f_{1}^{\prime}\left(x_{0}\right)-f_{2}^{\prime}\left(x_{0}\right)$. 
(36) For all $a, f, x_{0}$ such that $f$ is differentiable in $x_{0}$ holds $a f$ is differentiable in $x_{0}$ and $(a f)^{\prime}\left(x_{0}\right)=a \cdot f^{\prime}\left(x_{0}\right)$.

(37) Let given $f_{1}, f_{2}, x_{0}$. Suppose $f_{1}$ is differentiable in $x_{0}$ and $f_{2}$ is differentiable in $x_{0}$. Then $f_{1} f_{2}$ is differentiable in $x_{0}$ and $\left(f_{1} f_{2}\right)^{\prime}\left(x_{0}\right)=$ $\left(f_{2}\right)_{x_{0}} \cdot f_{1}{ }^{\prime}\left(x_{0}\right)+\left(f_{1}\right)_{x_{0}} \cdot f_{2}{ }^{\prime}\left(x_{0}\right)$.

(38) For all $f, Z$ such that $Z \subseteq \operatorname{dom} f$ and $f \nmid Z=\operatorname{id}_{Z}$ holds $f$ is differentiable on $Z$ and for every $x$ such that $x \in Z$ holds $\left(f_{\lceil Z}^{\prime}\right)_{x}=1_{\mathbb{C}}$.

(39) Let given $f_{1}, f_{2}, Z$. Suppose $Z \subseteq \operatorname{dom}\left(f_{1}+f_{2}\right)$ and $f_{1}$ is differentiable on $Z$ and $f_{2}$ is differentiable on $Z$. Then $f_{1}+f_{2}$ is differentiable on $Z$ and for every $x$ such that $x \in Z$ holds $\left(\left(f_{1}+f_{2}\right)^{\prime}{ }_{Y Z}\right)_{x}=f_{1}{ }^{\prime}(x)+f_{2}{ }^{\prime}(x)$.

(40) Let given $f_{1}, f_{2}, Z$. Suppose $Z \subseteq \operatorname{dom}\left(f_{1}-f_{2}\right)$ and $f_{1}$ is differentiable on $Z$ and $f_{2}$ is differentiable on $Z$. Then $f_{1}-f_{2}$ is differentiable on $Z$ and for every $x$ such that $x \in Z$ holds $\left(\left(f_{1}-f_{2}\right)^{\prime}{ }_{Y Z}\right)_{x}=f_{1}{ }^{\prime}(x)-f_{2}{ }^{\prime}(x)$.

(41) Let given $a, f, Z$. Suppose $Z \subseteq \operatorname{dom}(a f)$ and $f$ is differentiable on $Z$. Then $a f$ is differentiable on $Z$ and for every $x$ such that $x \in Z$ holds $\left((a f)^{\prime}{ }_{\mid Z}\right)_{x}=a \cdot f^{\prime}(x)$.

(42) Let given $f_{1}, f_{2}, Z$. Suppose $Z \subseteq \operatorname{dom}\left(f_{1} f_{2}\right)$ and $f_{1}$ is differentiable on $Z$ and $f_{2}$ is differentiable on $Z$. Then $f_{1} f_{2}$ is differentiable on $Z$ and for every $x$ such that $x \in Z$ holds $\left(\left(f_{1} f_{2}\right)^{\prime}{ }^{\prime}\right)_{x}=\left(f_{2}\right)_{x} \cdot f_{1}{ }^{\prime}(x)+\left(f_{1}\right)_{x} \cdot f_{2}{ }^{\prime}(x)$.

(43) If $Z \subseteq \operatorname{dom} f$ and $f$ is a constant on $Z$, then $f$ is differentiable on $Z$ and for every $x$ such that $x \in Z$ holds $\left(f_{\uparrow Z}^{\prime}\right)_{x}=0_{\mathbb{C}}$.

(44) Suppose $Z \subseteq \operatorname{dom} f$ and for every $x$ such that $x \in Z$ holds $f_{x}=a \cdot x+b$. Then $f$ is differentiable on $Z$ and for every $x$ such that $x \in Z$ holds $\left(f_{\uparrow Z}^{\prime}\right)_{x}=a$.

(45) For every complex number $x_{0}$ such that $f$ is differentiable in $x_{0}$ holds $f$ is continuous in $x_{0}$.

(46) If $f$ is differentiable on $X$, then $f$ is continuous on $X$.

(47) If $f$ is differentiable on $X$ and $Z \subseteq X$, then $f$ is differentiable on $Z$.

(48) If $s_{1}$ is convergent, then $\left|s_{1}\right|$ is convergent.

(49) If $f$ is differentiable in $x_{0}$, then there exists $R$ such that $R_{0_{\mathbb{C}}}=0_{\mathbb{C}}$ and $R$ is continuous in $0_{\mathbb{C}}$.

\section{REFERENCES}

[1] Agnieszka Banachowicz and Anna Winnicka. Complex sequences. Formalized Mathematics, 4(1):121-124, 1993.

[2] Grzegorz Bancerek. The fundamental properties of natural numbers. Formalized Mathematics, 1(1):41-46, 1990.

[3] Grzegorz Bancerek. The ordinal numbers. Formalized Mathematics, 1(1):91-96, 1990.

[4] Czesław Byliński. The complex numbers. Formalized Mathematics, 1(3):507-513, 1990.

[5] Czesław Byliński. Functions and their basic properties. Formalized Mathematics, 1(1):5565, 1990. 
[6] Czesław Byliński. Functions from a set to a set. Formalized Mathematics, 1(1):153-164, 1990.

[7] Czesław Byliński. Partial functions. Formalized Mathematics, 1(2):357-367, 1990.

[8] Krzysztof Hryniewiecki. Basic properties of real numbers. Formalized Mathematics, 1(1):35-40, 1990.

[9] Jarosław Kotowicz. Convergent sequences and the limit of sequences. Formalized Mathematics, 1(2):273-275, 1990.

[10] Jarosław Kotowicz. Monotone real sequences. Subsequences. Formalized Mathematics, $1(3): 471-475,1990$.

[11] Jarosław Kotowicz. Partial functions from a domain to a domain. Formalized Mathematics, 1(4):697-702, 1990.

[12] Jarosław Kotowicz. Real sequences and basic operations on them. Formalized Mathematics, 1(2):269-272, 1990.

[13] Takashi Mitsuishi, Katsumi Wasaki, and Yasunari Shidama. Property of complex sequence and continuity of complex function. Formalized Mathematics, 9(1):185-190, 2001.

[14] Adam Naumowicz. Conjugate sequences, bounded complex sequences and convergent complex sequences. Formalized Mathematics, 6(2):265-268, 1997.

[15] Yasunari Shidama and Artur Korniłowicz. Convergence and the limit of complex sequences. Series. Formalized Mathematics, 6(3):403-410, 1997.

[16] Andrzej Trybulec. Binary operations applied to functions. Formalized Mathematics, 1(2):329-334, 1990.

[17] Zinaida Trybulec. Properties of subsets. Formalized Mathematics, 1(1):67-71, 1990.

[18] Edmund Woronowicz. Relations and their basic properties. Formalized Mathematics, 1(1):73-83, 1990.

[19] Edmund Woronowicz. Relations defined on sets. Formalized Mathematics, 1(1):181-186, 1990.

Received November 4, 2008 